Physics

Physics Research Publications

Purdue University

Year 2010

\title{
Possibility of measuring the thermal Casimir interaction between a plate and a cylinder attached to a micromachined oscillator
}
R. S. Decca
E. Fischbach
G. L. Klimchitskaya
D. E. Krause
D. Lopez
V. M. Mostepanenko

This paper is posted at Purdue e-Pubs.

http://docs.lib.purdue.edu/physics_articles/1226 


\title{
Possibility of measuring the thermal Casimir interaction between a plate and a cylinder attached to a micromachined oscillator
}

\author{
R. S. Decca, ${ }^{1}$ E. Fischbach, ${ }^{2}$ G. L. Klimchitskaya, ${ }^{3}$ D. E. Krause, ${ }^{2,4}$ D. López, ${ }^{5}$ and V. M. Mostepanenko ${ }^{6}$ \\ ${ }^{1}$ Department of Physics, Indiana University-Purdue University Indianapolis, Indianapolis, Indiana 46202, USA \\ ${ }^{2}$ Department of Physics, Purdue University, West Lafayette, Indiana 47907, USA \\ ${ }^{3}$ North-West Technical University, Millionnaya Street 5, St. Petersburg 191065, Russia \\ ${ }^{4}$ Physics Department, Wabash College, Crawfordsville, Indiana 47933, USA \\ ${ }^{5}$ Center for Nanoscale Materials, Argonne National Laboratory, Argonne, Illinois 60439, USA \\ ${ }^{6}$ Noncommercial Partnership “Scientific Instruments," Tverskaya Street 11, Moscow 103905, Russia
}

(Received 27 August 2010; published 19 November 2010)

\begin{abstract}
We investigate the possibility of measuring the thermal Casimir force and its gradient in the configuration of a plate and a microfabricated cylinder attached to a micromachined oscillator. The Lifshitz-type formulas in this configuration are derived using the proximity force approximation. The accuracy of the obtained expressions is determined from a comparison with exact results available in ideal metal case. Computations of the thermal correction to both the Casimir force and its gradient are performed in the framework of different theoretical approaches proposed in the literature. The correction to the Casimir force and its gradient due to lack of parallelism of the plate and cylinder is determined using the nonmultiplicative approach. The error introduced in the theory due to the finite length of the cylinder is estimated. We propose that both static and dynamic experiments measuring the thermal Casimir interaction between a cylinder and a plate using a micromachined oscillator can shed additional light on the thermal Casimir force problem. Specifically, it is shown that the static experiment is better adapted for the measurement of thermal effects.
\end{abstract}

DOI: 10.1103/PhysRevA.82.052515

PACS number(s): 31.30.jh, 12.20.Ds, 12.20.Fv, 77.22.Ch

\section{INTRODUCTION}

The Casimir effect which was predicted more than 60 years ago [1] has recently attracted much theoretical and experimental attention (see the monographs [2-6] for an overview of the subject). The Casimir force originates from quantum fluctuations of the electromagnetic field. Thus, it is of the same origin as the van der Waals force, but acts at larger separations between the interacting surfaces where relativistic retardation effects become significant. The unified theory of both the van der Waals and the Casimir forces was developed by Lifshitz [7,8]. It describes the free energy and force of the van der Waals and Casimir interaction using the frequency-dependent dielectric permittivity of interacting bodies.

The Casimir force acting between two neutral surfaces becomes dominant at separations below $1 \mu \mathrm{m}$. Until the past decade there was a lack of experimental information about this quantum phenomenon, but this situation has changed in recent years due to rapid progress in nanotechnology. Following two landmark experiments $[9,10]$, many measurements of the Casimir and Casimir-Polder (atom-wall) forces have been performed with metal, semiconductor, and dielectric surfaces (a review of all experiments can be found in Ref. [11]).

The experimental and theoretical investigation of the Casimir force at nonzero temperature produced an unexpected result. It turned out that if conduction electrons in metal plates with perfect crystal lattices are described by the dissipative Drude model, this results in a violation of the Nernst heat theorem in the Lifshitz theory [6,11-13]. The same takes place for dielectric-type semiconductor and dielectric test bodies if free charge carriers, which are unavoidably present at nonzero temperature, are described by the Drude-type contribution to the dielectric permittivity $[6,11,14-16]$. The parameter range, where a violation occurs, was recently rederived and extended also to the geometry of a sphere above a plate [17]. It was demonstrated that for metals with impurities the Nernst heat theorem in the Lifshitz theory is preserved [18-21]. However, in the case of metals with perfect crystal structure (which is the basic model used in condensed-matter physics) and for dielectrics, a satisfactory solution was not found. This even resulted in the attempts [22,23] to modify the Lifshitz theory, and this led to a controversial discussion in the literature $[6,11,24-31]$. From the experimental side, it was shown that data exclude the Lifshitz theory if the role of conduction electrons is taken into account by means of the dissipative Drude model. This was repeatedly demonstrated for metal [32-36], semiconductor [37,38], and dielectric [39,40] test bodies.

All modern experiments measuring the Casimir force (with the single exception of the experiment of Ref. [41], which was burdened by rather large error of about 15\%) were performed using the configuration of a sphere above a plate. Bearing in mind that contradictions between Lifshitz theory and thermodynamics and experimental data discussed earlier are of great concern for the foundations of quantum statistical physics [42], it is worthwhile to consider the possibilities of alternative experiments. One more configuration of great promise that was discussed in the literature $[43,44]$ is a cylinder parallel to a plane plate. In some sense it is intermediate between the configurations of two parallel plates and a sphere above a plate because it preserves some advantages of the latter while making the problem of preserving the parallelism less difficult than for two plates. An additional advantage is that the Casimir force in the cylinder-plate geometry was recently calculated exactly in an ideal metal case [45-47]. This allows one to reliably estimate possible errors introduced from the use of the proximity force approximation. 
The original proposal [44] considered a relatively large cylinder with length $L=2 \mathrm{~cm}$ and diameter $2 R=6.35 \mathrm{~mm}$ separated from the plate with a wide gap $a>1 \mu \mathrm{m}$. It has been shown $[48,49]$, however, that for metal-coated spherical lenses with centimeter-size curvature radii, the electrostatic calibration of the Casimir apparatus meets serious difficulties. Specifically, the residual potential difference may become dependent on separation, and the force-distance relation for the electric force deviates from the form predicted by classical electrodynamics in a sphere-plane geometry. (This does not happen in the experiments of Refs. [32-36] dealing with spheres of about $150 \mu \mathrm{m}$ in radius interacting with the plate of an oscillator.) Similar anomalies were reported in Ref. [50] for the configuration of a cylindrical lens above a plate with lens parameters $L=4 \mathrm{~mm}, R=12 \mathrm{~mm}$. In Ref. [51] this anomalous behavior of the electric force was attributed to unavoidable deviations of the mechanically polished and ground surfaces from the perfect spherical shape assumed in elecrodynamical calculations. As recognized in Ref. [50], the same effect might be responsible for the calibration problems arising in the case of a centimeter-size cylinder above a plate. Because of this, the consideration of much smaller cylinders seems to be preferable.

In this article we investigate the possibility of combining the advantage of high precision provided by the setup of a micromachined oscillator and of a cylinder-plate configuration with a cylinder radius of about $100 \mu \mathrm{m}$. For this purpose we consider the thermal Casimir interaction between a metallized plate and a microfabricated metal-coated cylinder attached to a micromachined oscillator. The potentially increased precision of force measurements combined with the significantly decreased sizes of cylinders, as compared with Refs. [44,50], requires computations of the thermal Casimir force with a more sophisticated account of such factors as the lack of parallelism of the cylinder and plate and the finiteness of the length of a cylinder. Hence, we derive the Lifshitz-type formulas for the Casimir force and for its gradient for a cylinder and a plate made of real materials using the proximity force approximation (PFA). The accuracy of the results is determined by a comparison with the exact expressions available for a cylinder and a plate made of an ideal metal [46]. Computations of the thermal correction to the Casimir force and to its gradient using the resulting formulas are performed in the framework of different theoretical approaches proposed in the literature. The correction to the Casimir force and its gradient due to the lack of parallelism of a plate and a cylinder is determined in the nonmultiplicative way. The error due to the finiteness of the length of a cylinder is estimated using the results obtained with the help of world-line numerics [52]. Both static and dynamic experiments measuring the Casimir force and its gradient in the cylinder-plate configuration using a micromachined oscillator are proposed. It is shown that the static experiment is better adapted for the measurement of the thermal correction to the Casimir force.

This article is organized as follows. In Sec. II we derive the Lifshitz-type formulas for a cylinder-plate configuration. Section III is devoted to the computations of thermal corrections to the Casimir force and to its gradient. The correction due to the lack of parallelism of a cylinder and a plate is found in Sec. IV. In Sec. V we estimate the error introduced by the finiteness of a cylinder. The proposed experiments are discussed in Sec. VI. Section VII contains our conclusions and discussion.

\section{LIFSHITZ-TYPE FORMULAS FOR A CYLINDER ABOVE A PLATE MADE OF REAL MATERIALS}

Let us consider the upper surface of a metallized plate nearest to the cylinder as a coordinate plane $z=0$. For convenience of calculations, in Secs. II-V we consider the cylinder arranged above the plate. All the results obtained, however, refer equally well to the cylinder arranged below the plate (see Sec. VI). In real experiments, the cylinders used may have elliptical cross sections. Elliptical cylinders can be considered using the same methods as applied in what follows to circular cylinders. The cylinder axis (coinciding with the $y$ axis) is parallel to the plane $z=0$ at a separation distance $R+a$, where $R$ is the cylinder radius and $a$ is the minimum separation between the cylinder surface and the plate. The lower half of the cylinder surface is described by the equation

$$
z(x)=R+a-\sqrt{R^{2}-x^{2}} .
$$

We approximately replace this half with a set of thick plane plates extended along the $y$ axis made of the same material as the cylinder and parallel to the oscillator plate. Let us suppose that the Casimir pressure $P((z, x), T)$ between each of these plates and its projection on the plane $z=0$ at temperature $T$ is known. According to the Derjaguin method [6,53], under the condition $a \ll R$ the Casimir force acting between a cylinder and a plate can be approximately calculated as

$$
F(a, T)=\int_{\sigma} P(z(x), T) d x d y,
$$

where integration holds over the projection of a cylinder on the plane $z=0$. Incorporating some additional assumptions, the Derjaguin method was reformulated as the PFA [54] and widely used in the literature for the calculation of the Casimir force between a sphere and a plate (different versions of this method are discussed in Refs. [6,55]).

We assume that the material of the plate and the cylinder $\mathrm{Au}$ ) is characterized by the frequency-dependent dielectric permittivity $\varepsilon(\omega)$. (In fact, as described in Sec. VI, both the plate and the cylinder are coated with an Au layer with sufficient thickness that they can be considered as made of bulk Au.) The Casimir pressure between the plates at a separation $z(x)$ at temperature $T$ is given by the Lifshitz formula:

$$
\begin{aligned}
P(z(x), T)= & -\frac{k_{B} T}{\pi} \sum_{l=0}^{\infty} \int_{0}^{\infty} q_{l} k_{\perp} d k_{\perp} \\
& \times \sum_{\alpha} \frac{r_{\alpha}^{2} e^{-2 q_{l} z(x)}}{1-r_{\alpha}^{2} e^{-2 q_{l} z(x)}} .
\end{aligned}
$$

Here, $k_{B}$ is the Boltzmann constant, $k_{\perp}$ is the projection of the wave vector on the plane $z=0, q_{l}=\left(k_{\perp}^{2}+\xi_{l}^{2} / c^{2}\right)^{1 / 2}$, and $\xi_{l}=2 \pi k_{B} T l / \hbar$ with $l=0,1,2, \ldots$ are the Matsubara frequencies. The primed summation means that the term with $l=0$ is multiplied by $1 / 2$. The reflection coefficients $r_{\alpha}$ for the two polarizations of the electromagnetic field (transverse 
magnetic with $\alpha=\mathrm{TM}$ and transverse electric with $\alpha=\mathrm{TE}$ ) are given by

$$
\begin{gathered}
r_{\mathrm{TM}}=r_{\mathrm{TM}}\left(i \xi_{l}, k_{\perp}\right)=\frac{\varepsilon_{l} q_{l}-k_{l}}{\varepsilon_{l} q_{l}+k_{l}}, \\
r_{\mathrm{TE}}=r_{\mathrm{TE}}\left(i \xi_{l}, k_{\perp}\right)=\frac{q_{l}-k_{l}}{q_{l}+k_{l}},
\end{gathered}
$$

where $k_{l}=\left[k_{\perp}^{2}+\varepsilon_{l} \xi_{l}^{2} / c^{2}\right]^{1 / 2}$ and $\varepsilon_{l}=\varepsilon\left(i \xi_{l}\right)$. Notice that Eq. (3) can be identically represented as

$$
\begin{aligned}
P(z(x), T)= & -\frac{k_{B} T}{\pi} \sum_{l=0}^{\infty} \int_{0}^{\infty} q_{l} k_{\perp} d k_{\perp} \\
& \times \sum_{n=1}^{\infty} \sum_{\alpha} r_{\alpha}^{2 n} e^{-2 n q_{l} z(x)} .
\end{aligned}
$$

Now we substitute Eq. (5) into Eq. (2) and arrive at the expression

$$
\begin{aligned}
F(a, T)= & -\frac{2 k_{B} T L}{\pi} \sum_{l=0}^{\infty} \int_{0}^{\infty} q_{l} k_{\perp} d k_{\perp} \\
& \times \sum_{n=1}^{\infty}\left(r_{\mathrm{TM}}^{2 n}+r_{\mathrm{TE}}^{2 n}\right) \int_{0}^{R} d x e^{-2 n q_{l} z(x)},
\end{aligned}
$$

where $L$ is the length of the cylinder which is assumed to be infinitely large. Using Eq. (1) we replace the integration with respect to $x$ with an integration with respect to $z$. We then introduce two new integration variables, $u=(z-a) / R$ and $v=2 q_{l} a$, and rewrite Eq. (6) in the form

$$
\begin{aligned}
F(a, T)= & -\frac{k_{B} T R L}{4 \pi a^{3}} \sum_{l=0}^{\infty} \int_{\tau l}^{\infty} v^{2} d v \\
& \times \sum_{n=1}^{\infty} e^{-n v}\left(r_{\mathrm{TM}}^{2 n}+r_{\mathrm{TE}}^{2 n}\right) \int_{0}^{1} d u \frac{(1-u) e^{-n \frac{R}{a} v u}}{\sqrt{1-(1-u)^{2}}} .
\end{aligned}
$$

Here, the dimensionless quantity $\tau$ is defined as $\tau=$ $4 \pi k_{B} T a /(\hbar c)$, and the reflection coefficients are expressed in terms of dimensionless variables $v$ and $\zeta_{l}=\xi_{l} / \omega_{c}=\tau l$, where $\omega_{c}=c /(2 a)$, in the following way:

$$
\begin{gathered}
r_{\mathrm{TM}}=r_{\mathrm{TM}}\left(v, \zeta_{l}\right)=\frac{\varepsilon_{l} v-\sqrt{v^{2}+\left(\varepsilon_{l}-1\right) \zeta_{l}^{2}}}{\varepsilon_{l} v+\sqrt{v^{2}+\left(\varepsilon_{l}-1\right) \zeta_{l}^{2}}}, \\
r_{\mathrm{TE}}=r_{\mathrm{TE}}\left(v, \zeta_{l}\right)=\frac{v-\sqrt{v^{2}+\left(\varepsilon_{l}-1\right) \zeta_{l}^{2}}}{v+\sqrt{v^{2}+\left(\varepsilon_{l}-1\right) \zeta_{l}^{2}}} .
\end{gathered}
$$

Within the PFA, only the term of leading order in $a / R$ is physically meaningful. Because of this the integral with respect to $u$ in Eq. (7) can be evaluated as

$$
\begin{array}{rl}
\int_{0}^{1} & d u \frac{(1-u)}{\sqrt{1-(1-u)^{2}}} e^{-n \frac{R}{a} v u} \\
& =\int_{0}^{1} d u e^{-n \frac{R}{a} v u}\left[\frac{1}{\sqrt{2 u}}-\frac{3}{4} \sqrt{\frac{u}{2}}+O\left(u^{3 / 2}\right)\right] \\
& =\sqrt{\frac{\pi a}{2 n v R}}+O\left(\left(\frac{a}{R}\right)^{3 / 2}\right) .
\end{array}
$$

Substituting Eq. (9) into Eq. (7), we obtain

$$
\begin{aligned}
F(a, T)= & -\frac{k_{B} T L}{4 \sqrt{\pi} a^{2}} \sqrt{\frac{R}{2 a}} \sum_{l=0}^{\infty} \int_{\tau l}^{\infty} v^{3 / 2} d v \\
& \times \sum_{n=1}^{\infty} \frac{e^{-n v}}{\sqrt{n}}\left(r_{\mathrm{TM}}^{2 n}+r_{\mathrm{TE}}^{2 n}\right) .
\end{aligned}
$$

Using the definition of the polylogarithm function, the Lifshitz-type formula (10) describing the Casimir force for a cylinder in close proximity to a plate can be represented in the form

$$
\begin{aligned}
F(a, T)= & -\frac{k_{B} T L}{4 \sqrt{\pi} a^{2}} \sqrt{\frac{R}{2 a}} \sum_{l=0}^{\infty} \int_{\tau l}^{\infty} v^{3 / 2} d v \\
& \times\left[\operatorname{Li}_{1 / 2}\left(r_{\mathrm{TM}}^{2} e^{-v}\right)+\operatorname{Li}_{1 / 2}\left(r_{\mathrm{TE}}^{2} e^{-v}\right)\right] .
\end{aligned}
$$

This equation can be used in numerical computations of the Casimir force acting between a cylinder and a plate (see the next section).

In the case of a cylinder and a plate made of ideal metal, $r_{\mathrm{TM}}^{2}=r_{\mathrm{TE}}^{2}=1$. Then at zero temperature Eq. (11) takes the form

$$
F^{\mathrm{IM}}(a, 0)=-\frac{k_{B} T L}{2 \sqrt{\pi} a^{2}} \sqrt{\frac{R}{2 a}} \int_{0}^{\infty} d l \int_{\tau l}^{\infty} v^{3 / 2} d v \sum_{n=1}^{\infty} \frac{e^{-n v}}{\sqrt{n}}
$$

Introducing the new variable $\zeta=\tau l$, changing the order of integrations, and calculating the integrals with respect to $\zeta$ and $v$, one obtains

$$
F^{\mathrm{IM}}(a, 0)=-\frac{15 \hbar c L}{64 \pi a^{3}} \sqrt{\frac{R}{2 a}} \sum_{n=1}^{\infty} \frac{1}{n^{4}}=-\frac{\pi^{3} \hbar c L}{384 a^{3}} \sqrt{\frac{R}{2 a}} .
$$

Equation (13) coincides with the familiar expression for the configuration of an ideal metal cylinder above an ideal metal plate obtained using the PFA in Ref. [43] (see also Refs. $[6,44])$.

Both Eqs. (11) and (13) are approximate results. Bearing in mind that Eq. (11) can be used for the comparison of experimental data with theory, it is important to estimate how accurate it is. Fortunately, for an ideal metal cylinder above an ideal metal plate at zero temperature the exact analytical result for the two leading terms of the expansion of the Casimir force in powers of $a / R$ is available [46]:

$$
\begin{gathered}
F_{\mathrm{ex}}^{\mathrm{IM}}(a, 0)=-\frac{\pi^{3} \hbar c L}{384 \pi a^{3}} \sqrt{\frac{R}{2 a}}\left(1-C_{\mathrm{ex}} \frac{a}{R}\right), \\
C_{\mathrm{ex}}=\frac{4}{\pi^{2}}-\frac{7}{60} \approx 0.2886 .
\end{gathered}
$$

This result was confirmed by means of numerical computations in Ref. [47]. From the comparison of Eqs. (13) and (14) it can be seen that under the condition $a \ll R$ the deviation of the PFA result from the exact result does not exceed $0.3 a / R$. For a typical experimental value of $a / R \approx 10^{-3}$ the error in using the PFA turns out to be of about $0.03 \%$. For the configuration of an ideal metal sphere above an ideal metal plate at temperature $k_{B} T \gg \hbar c / R$ it was shown [56] that in the zeroth order of $a / R$ the PFA leads to exact results for the zero-temperature contribution to the Casimir force 
and, separately, for the thermal correction to it. It was also demonstrated that at zero temperature for a sphere and a plate made of real metals the deviation of the PFA results from the exact results is smaller than for a sphere and a plate made of an ideal metal [57-60]. Finally, exact computations of the thermal Casimir force at nonzero temperature in a sphere-plate configuration performed with different $a / R$ show that the exact results approach the PFA results when $a / R \rightarrow 0$ [60-62]. All the preceding suggests that for the configuration of a real metal cylinder above a real metal plate, the error of the Lifshitz-type Eq. (11) obtained using the PFA is of about the same, $0.3 a / R$, as holds for ideal metal bodies at $T=0$.

The Lifshitz-type formula for the gradient of the Casimir force can be obtained in a similar way to Eq. (11). For this purpose we differentiate Eq. (6) with respect to $a$ taking into account Eq. (1):

$$
\begin{aligned}
F^{\prime}(a, T) \equiv & \frac{\partial F(a, T)}{\partial a}=\frac{4 k_{B} T L}{\pi} \sum_{l=0}^{\infty} \int_{0}^{\infty} q_{l}^{2} k_{\perp} d k_{\perp} \\
& \times \sum_{n=1}^{\infty} n\left(r_{\mathrm{TM}}^{2 n}+r_{\mathrm{TE}}^{2 n}\right) \int_{0}^{R} d x e^{-2 n q_{l} z(x)} .
\end{aligned}
$$

Dealing with this equation as with Eq. (6), in the leading order of $a / R$ one obtains

$$
\begin{aligned}
\frac{\partial F(a, T)}{\partial a}= & \frac{k_{B} T L}{4 \sqrt{\pi} a^{3}} \sqrt{\frac{R}{2 a}} \sum_{l=0}^{\infty} \int_{\tau l}^{\infty} v^{5 / 2} d v \\
& \times \sum_{n=1}^{\infty} \sqrt{n} e^{-n v}\left(r_{\mathrm{TM}}^{2 n}+r_{\mathrm{TE}}^{2 n}\right) .
\end{aligned}
$$

Using the definition of the polylogarithm function results in

$$
\begin{aligned}
\frac{\partial F(a, T)}{\partial a}= & \frac{k_{B} T L}{4 \sqrt{\pi} a^{3}} \sqrt{\frac{R}{2 a}} \sum_{l=0}^{\infty} \int_{\tau l}^{\infty} v^{5 / 2} d v \\
& \times\left[\mathrm{Li}_{-1 / 2}\left(r_{\mathrm{TM}}^{2} e^{-v}\right)+\mathrm{Li}_{-1 / 2}\left(r_{\mathrm{TE}}^{2} e^{-v}\right)\right] .
\end{aligned}
$$

For an ideal metal cylinder above an ideal metal plate at zero temperature, Eq. (17) leads to the result

$$
\frac{\partial F^{\mathrm{IM}}(a, 0)}{\partial a}=\frac{7 \pi^{3} \hbar c L}{768 a^{4}} \sqrt{\frac{R}{2 a}},
$$

which is also immediately obtainable by differentiation of Eq. (13) with respect to $a$.

In the high-temperature (large-separation) limit only the zero-frequency terms of Eqs. (11) and (17) contribute to the result. Specifically, for the ideal metal case, one obtains from Eq. (11)

$$
\begin{aligned}
F^{\mathrm{IM}}(a, T) & =-\frac{k_{B} T L}{4 \sqrt{\pi} a^{2}} \sqrt{\frac{R}{2 a}} \int_{0}^{\infty} v^{3 / 2} \operatorname{Li}_{1 / 2}\left(e^{-v}\right) d v \\
& =-\frac{3 \zeta(3) k_{B} T L}{16 a^{2}} \sqrt{\frac{R}{2 a}},
\end{aligned}
$$

where $\zeta(z)$ is the Riemann $\zeta$ function. For the gradient of the Casimir force, this results in

$$
\frac{\partial F^{\mathrm{IM}}(a, T)}{\partial a}=\frac{15 \zeta(3) k_{B} T L}{32 a^{3}} \sqrt{\frac{R}{2 a}} .
$$

For real metals the asymptotic behavior at high temperature depends on the assumed model of dielectric properties (see the next section).

Although the immediately planned experiments are for metallic test bodies, the case of a dielectric cylinder above a dielectric plate is also of some experimental interest. For dielectrics with a finite dielectric permittivity at zero frequency, $\varepsilon(0)=\varepsilon_{0}$, the high-temperature behavior of the Casimir force is simply obtainable from Eqs. (10) and (11). In this case, at zero frequency we have

$$
r_{\mathrm{TM}}(0, v)=\frac{\varepsilon_{0}-1}{\varepsilon_{0}+1}=r_{0}, \quad r_{\mathrm{TE}}(0, v)=0 .
$$

The zero-frequency term of Eq. (10) then takes the form

$F^{\text {diel }}(a, T)=-\frac{k_{B} T L}{8 \sqrt{\pi} a^{2}} \sqrt{\frac{R}{2 a}} \sum_{n=1}^{\infty} \frac{r_{0}^{2 n}}{\sqrt{n}} \int_{0}^{\infty} v^{3 / 2} e^{-n v} d v$.

Calculating the integral with respect to $v$, the high-temperature behavior of the Casimir force between a dielectric plate and dielectric cylinder is obtained:

$$
\begin{aligned}
F^{\text {diel }}(a, T) & =-\frac{3 k_{B} T L}{32 a^{2}} \sqrt{\frac{R}{2 a}} \sum_{n=1}^{\infty} \frac{r_{0}^{2 n}}{n^{3}} \\
& =-\frac{3 k_{B} T L}{32 a^{2}} \sqrt{\frac{R}{2 a}} \operatorname{Li}_{3}\left(r_{0}^{2}\right) .
\end{aligned}
$$

The same equation, where $r_{0}^{2}$ is replaced with $r_{0}$, describes the high-temperature Casimir force acting between a dielectric plate and metallic cylinder (or, equivalently, metallic plate and dielectric cylinder). The latter result does not depend on the model used for the description of dielectric properties of the metal.

\section{COMPUTATIONS OF THE THERMAL CORRECTION TO THE CASIMIR FORCE BETWEEN A CYLINDER AND A PLATE COATED WITH GOLD}

Here, we use Eqs. (11) and (17) for computations of the relative thermal corrections to the Casimir force and to the gradient of the Casimir force defined as

$$
\begin{gathered}
\delta_{T}^{(1)}=\frac{F(a, T=300 K)-F(a, 0)}{F(a, T=300 K)}, \\
\delta_{T}^{(2)}=\frac{F^{\prime}(a, T=300 K)-F^{\prime}(a, 0)}{F^{\prime}(a, T=300 K)} .
\end{gathered}
$$

This requires knowledge of the complete data for the dielectric permittivity of Au along the imaginary frequency axis. Usually these are found using the dispersion relation

$$
\varepsilon(i \xi)=1+\frac{2}{\pi} \int_{0}^{\infty} \frac{\omega \operatorname{Im} \varepsilon(\omega)}{\omega^{2}+\xi^{2}} d \omega,
$$

where $\operatorname{Im} \varepsilon(\omega)$ is calculated from real and imaginary parts of the complex index of refraction $n$, tabulated, for example, in Ref. [63]. Keeping in mind that $\operatorname{Re} n(\omega)$ and $\operatorname{Im} n(\omega)$ are known only within a restricted frequency range (from 0.125 to $10000 \mathrm{eV}$ for Au in Ref. [63]), the problem of extrapolation of $\operatorname{Im} \varepsilon(\omega)$ to lower and higher frequencies arises (in fact, only the extrapolation to lower frequencies is of practical importance). According to the Drude model approach [6,11,18-21], the 
extrapolation of $\operatorname{Im} \varepsilon(\omega)$ to lower frequencies is performed using the imaginary part of the dielectric permittivity,

$$
\varepsilon_{D}(\omega)=1-\frac{\omega_{p}^{2}}{\omega(\omega+i \gamma)},
$$

where $\omega_{p}$ is the plasma frequency and $\gamma$ is the relaxation parameter (for Au we use [64] $\omega_{p}=9.0 \mathrm{eV}$ and $\gamma=0.035 \mathrm{eV}$ ).

According to the plasma model approach $[6,11,36,65,66]$, the dielectric permittivity can be expressed in the following oscillator form:

$$
\varepsilon(\omega)=1-\frac{\omega_{p}^{2}}{\omega^{2}}+\sum_{j=1}^{N} \frac{g_{j}}{\omega_{j}^{2}-\omega^{2}-i \gamma_{j} \omega},
$$

where the parameters of oscillators $\omega_{j} \neq 0, \gamma_{j}$, and $g_{j}$ are determined [36] from the best fit of $\operatorname{Im} \varepsilon(\omega)$ to the tabulated optical data for $n$ related to interband transitions of core electrons. As discussed in Sec. I, the Drude and the plasma model approaches lead to drastically different predictions for the thermal correction to the Casimir force in the sphere-plate geometry. Here, we perform all computations using both approaches in order to find additional evidence which could help to resolve this difference. Note that simple Drude and plasma models, which do not take interband transitions into account, were used in Ref. [43] to compute the Casimir interaction in a cylinder-plate configuration at nonzero temperature. Recently, an alternative approach was also proposed [67]. It aimed to determine $\varepsilon(i \xi)$ from both $\operatorname{Im} \varepsilon(\omega)$ and $\operatorname{Re} \varepsilon(\omega)$ within a frequency range where they are measured without using any extrapolations, but assuming the Drude-type behavior at zero frequency. It was shown [68], however, that large errors in the measurement data for $\operatorname{Re} \varepsilon(\omega)$ complicate the application of this approach in practical computations.

In Fig. 1 we present the computational results for the quantities $\delta_{T, D}^{(1)}$ (line 1) and $\delta_{T, D}^{(2)}$ (line 2) as functions of separation in the region from 0.1 to $5 \mu \mathrm{m}$. The computations were performed using the Drude model approach. As can be seen in Fig. 1, the thermal corrections to both the Casimir force and its gradient in a cylinder-plate configuration are negative over a wide range of separations. This is typical for a Drude model approach in the configurations of two

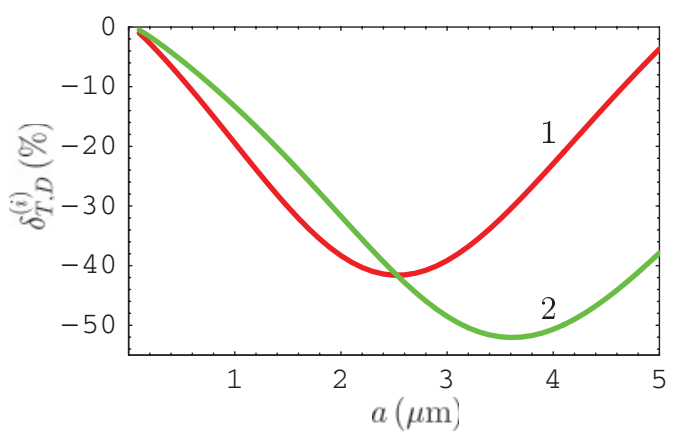

FIG. 1. (Color online) The relative thermal corrections to the Casimir force and its gradient in cylinder-plate configuration computed using the Drude model approach at $T=300 \mathrm{~K}$ (lines 1 and 2, respectively) as functions of separation in the region from 0.1 to $5 \mu \mathrm{m}$. parallel plates and a sphere above a plate as well. It should also be noted that the relative thermal correction achieves rather large values at short separation distances. Specifically, at experimentally relevant separations $a=150,200,300,500$, and $750 \mathrm{~nm}$ the thermal correction to the Casimir force is equal to $-1.8 \%,-2.7 \%,-4.6 \%,-8.6 \%$, and $-13.9 \%$, respectively. At the same respective separations the thermal correction to the gradient of the Casimir force achieves more moderate values $-0.1 \%,-1.6 \%,-2.9 \%,-5.6 \%$, and $-9.3 \%$. The magnitude of the relative thermal correction to the Casimir force $\left|\delta_{T, D}^{(1)}\right|$ achieves its maximum value $41.6 \%$ at $a=2.55 \mu \mathrm{m}$, while the maximum magnitude of the thermal correction to the gradient of the Casimir force $\left|\delta_{T, D}^{(2)}\right|=52 \%$ occurs at $a=3.6 \mu \mathrm{m}$. Thus, at large separation distances, dynamic experiments, where $\partial F / \partial a$ is a directly measured quantity, are most suitable for the detection of the thermal correction predicted within the Drude model approach.

In the high-temperature (large-separation) limit it is easy to obtain the analytic expression for the thermal Casimir force calculated using the Drude model approach. Taking into account that Eqs. (8) and (26) lead to $r_{\mathrm{TM}}(0, v)=1$ and $r_{\mathrm{TE}}(0, v)=0$, the contribution of the zero-frequency term in Eq. (11) is given by

$$
\begin{aligned}
F^{D}(a, T) & =-\frac{k_{B} T L}{8 \sqrt{\pi} a^{2}} \sqrt{\frac{R}{2 a}} \int_{0}^{\infty} v^{3 / 2} \operatorname{Li}_{1 / 2}\left(e^{-v}\right) d v \\
& =-\frac{3 \zeta(3) k_{B} T L}{32 a^{2}} \sqrt{\frac{R}{2 a}} .
\end{aligned}
$$

For the gradient of the Casimir force,

$$
\frac{\partial F^{\mathrm{D}}(a, T)}{\partial a}=\frac{15 \zeta(3) k_{B} T L}{64 a^{3}} \sqrt{\frac{R}{2 a}}
$$

is obtained.

We next present the computational results in the case where the plasma model approach is applied to describe metallic coatings on a cylinder and a plate. In Fig. 2(a) the computational results for the quantities $\delta_{T, p}^{(1)}$ (line 1) and $\delta_{T, p}^{(2)}$ (line 2) are shown as functions of separation in the region from 0.1 to $5 \mu \mathrm{m}$. In Fig. 2(b) the same results in the region from 0.1 to $1 \mu \mathrm{m}$ are shown at a larger scale. As for the configurations of two parallel plates and a sphere above a plate, the thermal correction for a cylinder above a plate is positive when the plasma model approach is used. As can be seen in Figs. 2(a) and 2(b), at short separations the relative thermal correction obtained within the plasma model approach is negligibly small. Thus, the correction to the Casimir force is equal to $0.016 \%, 0.024 \%, 0.044 \%, 0.13 \%$, and $0.38 \%$ at separations $a=150,200,300,500$, and $750 \mathrm{~nm}$, respectively. At the same respective separations, the thermal correction to the gradient of the Casimir force is equal to $0.0014 \%$, $0.0023 \%, 0.0046 \%, 0.012 \%$, and $0.029 \%$; that is, it is even less. This makes the observation of the thermal correction at short separations, as predicted by the plasma model, impossible in either the static or the dynamic experiments. However, at larger separations the thermal correction to the Casimir force computed using the plasma model approach quickly increases. It is equal to $0.9 \%, 7.2 \%$, and $46 \%$ at separations $a=1,2$, and $5 \mu \mathrm{m}$, respectively. At the same respective separations, the thermal correction to the gradient of the Casimir force is 

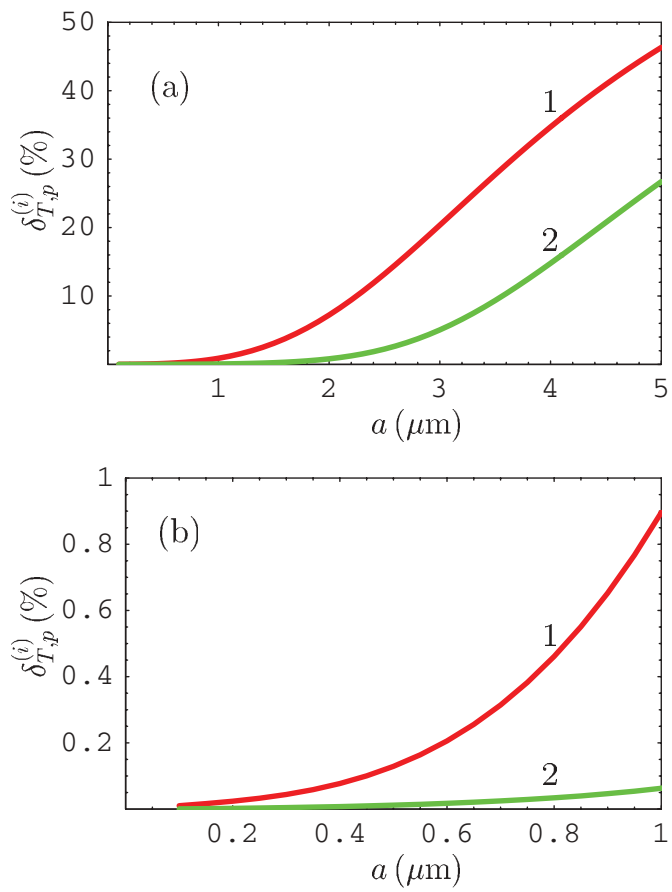

FIG. 2. (Color online) The relative thermal corrections to the Casimir force and its gradient in cylinder-plate configuration computed using the plasma model approach at $T=300 \mathrm{~K}$ (lines 1 and 2 , respectively) as functions of separation in the region (a) from 0.1 to $5 \mu \mathrm{m}$ and (b) from 0.1 to $1 \mu \mathrm{m}$.

equal to $0.063 \%, 0.82 \%$, and $26.7 \%$. Thus, within the plasma model approach the relative thermal correction to the Casimir force is always larger than that to the gradient of the Casimir force.

As in the case of the Drude model approach, one can obtain analytic expressions for the thermal Casimir force and its gradient at high temperature. From Eqs. (8) and (27), for the reflection coefficients at zero frequency, one obtains

$$
\begin{aligned}
r_{\mathrm{TM}}(0, v) & =1 \\
r_{\mathrm{TE}}(0, v)=\frac{\alpha v-\sqrt{\alpha^{2} v^{2}+1}}{\alpha v+\sqrt{\alpha^{2} v^{2}+1}} & =-\left(\alpha v-\sqrt{\alpha^{2} v^{2}+1}\right)^{2}
\end{aligned}
$$

where $\alpha=\omega_{c} / \omega_{p} \equiv \delta_{0} /(2 a)$. The quantity $\delta_{0}=c / \omega_{p}$ has the meaning of the skin depth in the frequency region of infrared optics. Equation (11) then leads to

$$
\begin{aligned}
F^{p}(a, T)= & -\frac{k_{B} T L}{8 \sqrt{\pi} a^{2}} \sqrt{\frac{R}{2 a}} \int_{0}^{\infty} v^{3 / 2} d v \\
& \times\left[\operatorname{Li}_{1 / 2}\left(e^{-v}\right)+\operatorname{Li}_{1 / 2}\left(r_{\mathrm{TE}}^{2}(0, v) e^{-v}\right)\right] .
\end{aligned}
$$

The integral of the first term on the right-hand side of Eq. (31) was calculated in Eq. (28). The integral of the second term can be calculated approximately with the help of perturbation theory in powers of the small parameter $\alpha$ (for Au $\delta_{0} \approx 22 \mathrm{~nm} \ll a$ ). Using Eq. (30), one obtains

$$
r_{\mathrm{TE}}^{2 n}(0, v)=1-4 n v \alpha+8 n^{2} v^{2} \alpha^{2}+O\left(\alpha^{3}\right)
$$

The second integral on the right-hand side of of Eq. (31) is then found using this equation:

$$
\begin{aligned}
& \int_{0}^{\infty} v^{3 / 2} \operatorname{Li}_{1 / 2}\left(r_{\mathrm{TE}}^{2}(0, v) e^{-v}\right) d v \\
& =\sum_{n=1}^{\infty} \frac{1}{\sqrt{n}} \int_{0}^{\infty} v^{3 / 2}\left(1-4 n \alpha v+8 n^{2} \alpha^{2} v^{2}\right) e^{-n v} d v \\
& =\frac{3 \sqrt{\pi} \zeta(3)}{4}\left(1-10 \alpha+70 \alpha^{2}\right) \\
& =\frac{3 \sqrt{\pi} \zeta(3)}{4}\left[1-5 \frac{\delta_{0}}{a}+\frac{35}{2}\left(\frac{\delta_{0}}{a}\right)^{2}\right] .
\end{aligned}
$$

By adding the contribution of the first integral in Eq. (31), we obtain the high-temperature asymptotic behavior of the Casimir force:

$$
F^{p}(a, T)=-\frac{3 \zeta(3) k_{B} T L}{16 a^{2}} \sqrt{\frac{R}{2 a}}\left[1-\frac{5}{2} \frac{\delta_{0}}{a}+\frac{35}{4}\left(\frac{\delta_{0}}{a}\right)^{2}\right] .
$$

The high-temperature asymptotic behavior for the gradient of the Casimir force is given by

$$
\frac{\partial F^{p}(a, T)}{\partial a}=\frac{15 \zeta(3) k_{B} T L}{32 a^{3}} \sqrt{\frac{R}{2 a}}\left[1-\frac{7}{2} \frac{\delta_{0}}{a}+\frac{63}{4}\left(\frac{\delta_{0}}{a}\right)^{2}\right] .
$$

From the comparison of Eqs. (34) and (35) with Eqs. (28) and (29), respectively, it can be seen that under the condition $a \ll R$ the main contributions to the high-temperature Casimir force and its gradient calculated using the plasma model approach are two times larger than the Casimir force and its gradient calculated using the Drude model approach. The same holds in the configuration of two parallel plates and for a sphere above a plate under the condition $a \ll R[6,11]$.

\section{CORRECTION DUE TO LACK OF PARALLELISM OF A PLATE AND A CYLINDER}

Experimentally, it is impossible to preserve the exact parallelism of a plate and a cylinder as was always assumed in the preceding calculations. Because of this, it is important to find the correction to the derived results which arises if there is some small angle $\theta \ll 1$ between the plate and the axis of a cylinder. For ideal metal bodies such a correction factor which depends on the mean separation between the cylinder and plate was found in Ref. [44]. The magnitude of the Casimir force between a real metal cylinder and a plate taking into account their nonparallelity was also estimated by multiplying the result obtained for parallel bodies by the same factor. Calculations show, however, that for real metal bodies corrections due to deviations from perfect geometry (for instance, due to surface roughness or due to a nonparallelity of two surfaces) are not of precisely multiplicative character $[6,11]$. Specifically, at short separations (typically at $a<200 \mathrm{~nm}$ ) the multiplicative approach may lead to large errors. Because of this, we here calculate the thermal Casimir force between a cylinder and a plate in a more inclusive way by applying the PFA to account not only for a cylindrical geometry but for a nonparallelity of a cylinder and a plate as well. 
We start with Eq. (6) for the Casimir force between a plate and a cylinder parallel to it. It is necessary to take into account that the cylinder is now inclined by a small angle $\theta$ with respect to the plane $z=0$. This means that thick plane plates extended along the $y$ axis, which are used in Sec. II as a substitution for a cylinder in the PFA, are also inclined at an angle $\theta$. We replace each of these inclined plates with a set of plates of area $d x d y$ parallel to the plane $z=0$. This is equivalent to replacing $z(x)$ in Eq. (6) with $z(x)+\theta y$ and subsequently averaging over the length of the cylinder. The Casimir force then takes the form

$$
\begin{aligned}
F(a, T, \theta)= & -\frac{2 k_{B} T}{\pi} \sum_{l=0}^{\infty} \int_{0}^{\infty} q_{l} k_{\perp} d k_{\perp} \\
& \times \sum_{n=1}^{\infty}\left(r_{\mathrm{TM}}^{2 n}+r_{\mathrm{TE}}^{2 n}\right) \int_{0}^{R} d x \int_{-L / 2}^{L / 2} d y e^{-2 n q_{l}[z(x)+\theta y]},
\end{aligned}
$$

where $a$ is now the mean minimum separation between the cylinder and the plate. At $\theta=0$ Eq. (36) coincides with Eq. (6).

The integration with respect to $y$ in Eq. (36) is trivial. We then introduce the other integration variables $u$ and $v$, defined in Sec. II, and perform calculations similar to those leading to Eq. (10). Finally, retaining only the leading-order contribution in the small parameter $a / R$, we arrive at

$$
\begin{aligned}
F(a, T, \theta)= & -\frac{k_{B} T L}{4 \sqrt{\pi} a^{2}} \sqrt{\frac{R}{2 a}} \sum_{l=0}^{\infty} \int_{\tau l}^{\infty} v^{3 / 2} d v \\
& \times \sum_{n=1}^{\infty} \frac{e^{-n v}}{\sqrt{n}}\left(r_{\mathrm{TM}}^{2 n}+r_{\mathrm{TE}}^{2 n}\right) \frac{\sinh \left(A_{\theta} n v\right)}{A_{\theta} n v},
\end{aligned}
$$

where one additional small parameter is defined as $A_{\theta}=$ $\theta L /(2 a)$. For ideal metals at zero temperature, Eq. (36) reads as

$$
\begin{aligned}
F^{\mathrm{IM}}(a, 0, \theta)= & -\frac{\hbar c L}{8 \pi \sqrt{\pi} a^{3}} \sqrt{\frac{R}{2 a}} \int_{0}^{\infty} v^{5 / 2} d v \\
& \times \sum_{n=1}^{\infty} \frac{e^{-n v}}{\sqrt{n}} \frac{\sinh \left(A_{\theta} n v\right)}{A_{\theta} n v} .
\end{aligned}
$$

After performing the integration and summation, Eq. (38) results in

$$
F^{\mathrm{IM}}(a, 0, \theta)=\kappa\left(A_{\theta}\right) F^{\mathrm{IM}}(a, 0),
$$

where $F^{\mathrm{IM}}(a, 0)$ holds for an ideal metal cylinder parallel to an ideal metal plate and is defined in Eq. (13), and the correction factor due to nonparallelity is given by

$$
\kappa\left(A_{\theta}\right)=\frac{1}{5 A_{\theta}}\left[\frac{1}{\left(1-A_{\theta}\right)^{5 / 2}}-\frac{1}{\left(1+A_{\theta}\right)^{5 / 2}}\right] .
$$

Note that this correction factor was first obtained in Ref. [44] by direct application of the PFA to an ideal metal cylinder inclined at an angle $\theta$ to an ideal metal plate. Here we have shown that Eq. (40) follows from a more general Lifshitz-type formula (38) which takes into account the nonmultiplicative effects due to nonparallelity of a plate and a cylinder. The correction factor $\kappa\left(A_{\theta}\right)$ can be also used at nonzero temperature in order to estimate the Casimir force between a cylinder and a plate under an angle $\theta$ by means of the approximate multiplicative approach:

$$
F^{\mathrm{m}}(a, T, \theta)=\kappa\left(A_{\theta}\right) F(a, T) .
$$

Here we estimate the accuracy of this approach at different angles and separation distances.

The same procedure as described earlier for the Casimir force can be used in order to find a nonmultiplicative effect of a nonzero angle between a cylinder and a plate on the gradient of the Casimir force. As a result, Eq. (16) is replaced with

$$
\begin{aligned}
\frac{\partial F(a, T)}{\partial a}= & \frac{k_{B} T L}{4 \sqrt{\pi} a^{3}} \sqrt{\frac{R}{2 a}} \sum_{l=0}^{\infty} \int_{\tau l}^{\infty} v^{5 / 2} d v \\
& \times \sum_{n=1}^{\infty} \sqrt{n} e^{-n v}\left(r_{\mathrm{TM}}^{2 n}+r_{\mathrm{TE}}^{2 n}\right) \frac{\sinh \left(A_{\theta} n v\right)}{A_{\theta} n v}
\end{aligned}
$$

Using Eqs. (11) and (37) we performed numerical computations of the quantity

$$
\kappa^{(\mathrm{nm})}\left(a, A_{\theta}, T\right)=\frac{F(a, T, \theta)}{F(a, T)}
$$

at different $a$ and $A_{\theta}$. This quantity takes into account the nonmultiplicative effects due to a nonzero angle between a cylinder and a plate. When compared with $\kappa\left(A_{\theta}\right)$, it allows us to determine the application region of the multiplicative approach. Note that $\kappa^{(\mathrm{nm})}$ depends on separation through the parameter $A_{\theta}$ and through the $a$-dependent quantity $\tau$ in the lower integration limits in Eqs. (11) and (37). As for the correction factor $\kappa$ defined in Eq. (40), it depends on separation only through the parameter $A_{\theta}$.

The computational results for $\kappa^{(\mathrm{nm})}$ (lines 1-6) and $\kappa$ (line 7) at $T=300 \mathrm{~K}$ are presented in Table I. Column 1 indicates separations $a$ at which the values of $\kappa^{(\mathrm{nm})}$ are computed (from 100 to $500 \mathrm{~nm}$ ). Columns 2, 3, 4, and 5 contain the results obtained for $A_{\theta}=0.01,0.05,0.1$, and 0.5 , respectively. Keeping in mind the definition of $A_{\theta}$, the value of $\theta=2 a A_{\theta} / L$ in each column changes with the change of separation. To determine the application region of the multiplicative approach, we assume that this approach should not lead to an error larger than the error due to the PFA. For the parameters of a cylinder we take $L=R=100 \mu \mathrm{m}$. Then, for instance, at $a=100 \mathrm{~nm}$ the relative error of the PFA is equal to $0.3 a / R=0.0003$ (see Sec. II). If $A_{\theta}=0.01$ (see column 2 of Table I), we have $\kappa-\kappa^{(\mathrm{nm})}(100 \mathrm{~nm})=0.00006$, that is, much less than the error of the PFA. Keeping in mind that at $a=$ $100 \mathrm{~nm}$ the inequality $A_{\theta} \leqslant 0.01$ is certainly valid at angles $\theta \leqslant 4^{\prime \prime}$, one arrives at the conclusion that with these angles between a cylinder and a plate the multiplicative approach is applicable. It is very hard, however, to ensure angles $\theta \leqslant 4^{\prime \prime} \approx 20 \mu \mathrm{rad}$ experimentally. Because of this, at short separations the nonmultiplicative approach should be used for the comparison between the experimental data and theory. With $A_{\theta}=0.01$ the multiplicative approach is applicable at larger separations, as well as at even larger angles. As one more example, we consider $A_{\theta}=0.05$ (column 3 of Table I). Here, $\kappa-\kappa^{(\mathrm{nm})}(100 \mathrm{~nm})=0.0015$, that is, larger than the error of the PFA at $a=100 \mathrm{~nm}$. Thus, the multiplicative approach is not applicable. However, at $a=500 \mathrm{~nm}$ the error of the PFA is equal to 0.0015 , whereas $\kappa-\kappa^{(\mathrm{nm})}(500 \mathrm{~nm})=0.0006$. Thus, 
TABLE I. The lines from 1 to 6 contain the values of the quantity $\kappa^{(\mathrm{nm})}$ quantifying the effect of the nonzero angle between a cylinder and a plate on the Casimir force within the nonmultiplicative approach. In line 7 the correction factor $\kappa$ incorporating the nonzero angle in a multiplicative way is presented. All results are computed at different values of the parameter $A_{\theta}=\theta L /(2 a)$.

\begin{tabular}{lcccc}
\hline \hline & $A_{\theta}=0.01$ & $A_{\theta}=0.05$ & $A_{\theta}=0.1$ & $A_{\theta}=0.5$ \\
\hline$\kappa^{(\mathrm{nm})}\left(100 \mathrm{~nm}, A_{\theta}\right)$ & 1.00020 & 1.0051 & 1.0207 & 1.7888 \\
$\kappa^{(\mathrm{nm})}\left(150 \mathrm{~nm}, A_{\theta}\right)$ & 1.00021 & 1.0053 & 1.0215 & 1.8230 \\
$\kappa^{(\mathrm{nm})}\left(200 \mathrm{~nm}, A_{\theta}\right)$ & 1.00022 & 1.0055 & 1.0221 & 1.8526 \\
$\kappa^{(\mathrm{nm})}\left(300 \mathrm{~nm}, A_{\theta}\right)$ & 1.00023 & 1.0057 & 1.0231 & 1.8997 \\
$\kappa^{(\mathrm{nm})}\left(400 \mathrm{~nm}, A_{\theta}\right)$ & 1.00023 & 1.0058 & 1.0237 & 1.9335 \\
$\kappa^{(\mathrm{nm})}\left(500 \mathrm{~nm}, A_{\theta}\right)$ & 1.00024 & 1.0060 & 1.0242 & 1.9585 \\
$\kappa\left(A_{\theta}\right)$ & 1.00026 & 1.0066 & 1.0267 & 2.1176 \\
\hline \hline
\end{tabular}

at this separation with the chosen parameters of a cylinder the multiplicative approach is applicable at $\theta \leqslant 1.7^{\prime}$. At larger angles the nonmultiplicative results in Eqs. (37) and (42) should be used to compute the Casimir force and its gradient with sufficient precision.

\section{ERROR DUE TO FINITE LENGTH OF A CYLINDER}

The Lifshitz-type formulas (11) and (17) were obtained under an assumption that the length $L$ of the cylinder is infinitely large. In fact, these formulas define the Casimir force and its gradient per unit length of a cylinder. Keeping in mind that in our experimental setup the length of the cylinder $L$ is not only finite but might be of the same order as the cylinder radius $R$, it is important to estimate the possible error due to the finiteness of $L$. Here, we solve this problem for an ideal metal cylinder above an ideal metal plate. The derived results can serve as a reasonable estimation for real metal bodies as well.

We start with the case when the plate is sufficiently large that the projection of the cylinder on the plane $(x, y)$ is situated within its interior [see Fig. 3(a)]. This is the case for the proposed experiment (see Sec. VI). It is assumed that the length of plane plates with an area $L d x$, which replace a cylinder when we apply the PFA to calculate the Casimir force (see Sec. II), is finite. As a result, two real edges of length $d x$ each are formed. Because of this, it is not legitimate to use either the original Casimir expression for the force per unit area $-\pi^{2} \hbar c /\left(240 a^{4}\right)$ or its Lifshitz counterpart (3) applicable to real metals in order to perform calculations in the framework of the PFA. Instead, we apply the expression [52] for the Casimir force acting between an infinite plate and a finite plate parallel to it of area $S$ with edge length $l$ spaced $z$ apart, obtained using the method of world-line numerics:

$$
F_{\text {ed }}(z, 0)=-\frac{\pi^{2} \hbar c S}{480 z^{4}}-\gamma_{a} \frac{\hbar c l}{z^{3}}
$$

This equation with $\gamma_{a}=5.23 \times 10^{-3}$ was obtained for a scalar field with Dirichlet boundary conditions on the plates at zero temperature. Keeping in mind applications to the electromagnetic field, the Casimir force acting between the

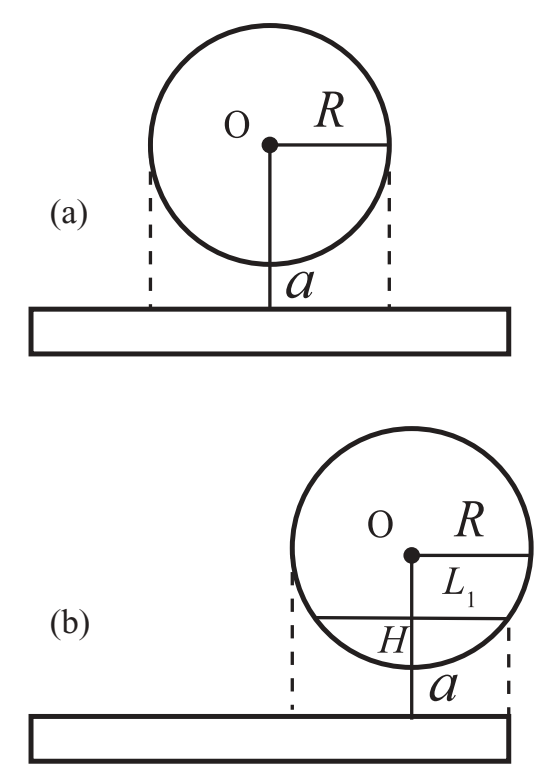

FIG. 3. The cylinder and the plate in cases when (a) the projection of the cylinder on the plane $(x, y)$ belongs to the interior of the plate and (b) part of the projection of the cylinder on the plane $(x, y)$ is beyond the edge of the plate.

partial plate of area $L d x$, replacing the cylindrical surface in the PFA, and the plane plate is given by

$$
d F_{\mathrm{ed}}^{\mathrm{IM}}(z, 0)=-\frac{\pi^{2} \hbar c L d x}{240 z^{4}}-4 \gamma_{a} \frac{\hbar c d x}{z^{3}}
$$

where $z=z(x)$ is defined in Eq. (1) and the length of real edges is $l=2 d x$.

The integration of Eq. (45) over the lower half of a cylinder in the leading order of the small parameter $a / R$ results in

$$
\begin{aligned}
F_{\mathrm{ed}}^{\mathrm{IM}}(a, 0) & =\int_{-R}^{R} d F_{\mathrm{ed}}^{\mathrm{IM}}(z, 0) \\
& =-\frac{\pi^{3} \hbar c L}{384 a^{3}} \sqrt{\frac{R}{2 a}}-\frac{3 \pi \gamma_{a} \hbar c}{a^{2}} \sqrt{\frac{R}{2 a}} \\
& =F^{\mathrm{IM}}(a, 0)\left(1+C_{\mathrm{ed}} \frac{a}{L}\right),
\end{aligned}
$$

where $F^{\mathrm{IM}}(a, 0)$ is defined in Eq. (13) and

$$
C_{\mathrm{ed}}=\frac{1152 \gamma_{a}}{\pi^{2}} \approx 0.610 .
$$

In a similar way, the gradient of the Casimir force accounting for edge effects is given by

$$
\frac{\partial F_{\mathrm{ed}}^{\mathrm{IM}}(a, 0)}{\partial a}=\frac{\partial F^{\mathrm{IM}}(a, 0)}{\partial a}\left(1+\tilde{C}_{\mathrm{ed}} \frac{a}{L}\right),
$$

where $\partial F^{\mathrm{IM}}(a, 0) / \partial a$ is defined in Eq. (18) and

$$
\tilde{C}_{\text {ed }}=\frac{5}{7} C_{\text {ed }} \approx 0.436 \text {. }
$$

We are now in a position to estimate the total relative error in the Casimir force and its gradient, originating from the application of the PFA to a cylinder of finite length. For the Casimir force this is a combination of the systematic errors due to the application of the PFA to an infinite cylinder, 
$-C_{\text {ex }} a / R$, estimated in Sec. II, and that due to the edge effects, $C_{\text {ed }} a / L$. Taking the most conservative approach, we consider these systematic errors as random quantities characterized by a uniform distribution. Then the total error of the Casimir force determined at a $95 \%$ confidence level can be obtained from the following statistical rule $[6,69]$ :

$$
\delta_{F}^{\mathrm{tot}}=\min \left(C_{\mathrm{ex}} \frac{a}{R}+C_{\mathrm{ed}} \frac{a}{L}, 1.1 \sqrt{C_{\mathrm{ex}}^{2} \frac{a^{2}}{R^{2}}+C_{\mathrm{ed}}^{2} \frac{a^{2}}{L^{2}}}\right) .
$$

Taking $R=L=100 \mu \mathrm{m}$, we find from Eq. (50) that $\delta_{F}^{\text {tot }} \approx$ $0.07 \%$ at $a=100 \mathrm{~nm}$ and $\delta_{F}^{\text {tot }} \approx 0.37 \%$ at $a=500 \mathrm{~nm}$.

To estimate the total error of the gradient of the Casimir force between a cylinder and a plate, one should use the exact expression following from Eq. (14)

$$
\begin{gathered}
\frac{\partial F_{\mathrm{ex}}^{\mathrm{IM}}(a, 0)}{\partial a}=\frac{7 \pi^{3} \hbar c L}{768 a^{4}} \sqrt{\frac{R}{2 a}}\left(1-\tilde{C}_{\mathrm{ex}} \frac{a}{L}\right), \\
\tilde{C}_{\mathrm{ex}}=\frac{5}{7} C_{\mathrm{ex}} \approx 0.2062 .
\end{gathered}
$$

This shows that the error in the application of the PFA to the gradient of the Casimir force between an infinite cylinder and a plate is less than in the application of this method to the force. Combining the errors $\tilde{C}_{\text {ex }} a / R$ from Eq. (51) and $\tilde{C}_{\text {ed }} a / L$ defined in Eq. (49) with the help of the same statistical rule (50), one obtains $\delta_{\partial F / \partial a}^{\text {tot }} \approx 0.05 \%$ and $0.26 \%$ at $a=100$ and $500 \mathrm{~nm}$, respectively.

In the dynamic experiments measuring the gradient of the Casimir force between a sphere and a plate by means of a micromachined oscillator [32-36] the Au-coated sphere was attached to the optical fiber. As a result, the projection of the part of the sphere near the point of attachment on the plane of an oscillator plate was situated beyond the plate edge. As was noted earlier, in the proposed experiment the cylinder is attached to an oscillator and the cylinder's projection on the plate belongs to its interior (see Sec. VI). However, in some other experimental configurations it may happen that the projection of the part of the cylinder is beyond the edge of the plate. We now consider the role of edge effects in the case when the projection of a cylinder axis on the plate is at distance $L_{1}<R$ from the edge of a plate [see Fig. 3(b)]. In this case one half of the cylinder is completely above the plate. As to the other half, only the portion $x \leqslant L_{1}$ is above the plate.

We next obtain the expression for the Casimir force taking into account edge effects,

$$
\tilde{F}_{\text {ed }}^{\mathrm{IM}}(a, 0)=\int_{-R}^{L_{1}} d F_{\mathrm{ed}}^{\mathrm{IM}}(z, 0)-\frac{2 \gamma_{a} \hbar c L}{(a+H)^{3}},
$$

where $d F_{\mathrm{ed}}^{\mathrm{IM}}(z, 0)$ is defined in Eq. (45), and $H=R-$ $\sqrt{R^{2}-L_{1}^{2}}$ [see Fig. 3(b)]. In the following we assume $L_{1}, H \gg a$. Here, the upper integration limit $L_{1}$ [instead of $R$ in Eq. (46)] takes into account that only part of the cylinder is above the plate. The second term on the right-hand side of Eq. (52) is due to the presence of an additional real boundary of length $L$. This boundary belongs to one of the partial plane plates, with area $L d x$ replacing a cylinder when the PFA is used which is situated at a height $a+H$ above the edge of an oscillator plate. Equation (52) can be equivalently presented in the form

$$
\tilde{F}_{\mathrm{ed}}^{\mathrm{IM}}(a, 0)=\int_{-R}^{R} d F_{\mathrm{ed}}^{\mathrm{IM}}(z, 0)-\int_{L_{1}}^{R} d F_{\mathrm{ed}}^{\mathrm{IM}}(z, 0)-\frac{2 \gamma_{a} \hbar c L}{(a+H)^{3}} .
$$

Performing the integrations and retaining only the leading terms in the small parameters $a / R$ and $a / H$, we arrive at the result

$$
\begin{aligned}
F_{\text {ed }}^{\mathrm{IM}}(a, 0)= & F^{\mathrm{IM}}(a, 0)\left[1+C_{\mathrm{ed}} \frac{a}{L}-f\left(\frac{L_{1}}{R}\right)\right. \\
& \left.\times \sqrt{\frac{a}{R}} \frac{a^{3}}{L_{1}^{3}}+\frac{768 \sqrt{2} \gamma_{a}}{\pi^{3}} \sqrt{\frac{a}{R}} \frac{a^{3}}{H^{3}}\right] .
\end{aligned}
$$

Here the following notations are introduced:

$$
f\left(\frac{L_{1}}{R}\right)=\frac{8 \sqrt{2}}{525 \pi} \frac{R^{4}}{L_{1}^{4}} f_{1}\left(\frac{L_{1}}{R}\right)+\frac{1536 \sqrt{2} \gamma_{a}}{5 \pi^{3}} \frac{R^{3}}{L L_{1}^{2}} f_{2}\left(\frac{L_{1}}{R}\right),
$$

where

$$
\begin{aligned}
f_{1}(z)= & 120-160 z^{2}+35 z^{4}+13 z^{7} \\
& +4 \sqrt{1-z^{2}}\left(30-27 z^{2}-z^{4}-2 z^{6}\right), \\
f_{2}(z)= & 4-5 z^{2}+z^{5}+\sqrt{1-z^{2}}\left(4-3 z^{2}-z^{4}\right) .
\end{aligned}
$$

If $L_{1}=R$ (i.e., the cylinder is completely over the plate), we have $f_{1}(1)=f_{2}(1)=f(1)=0$, and Eq. (54) reduces to Eq. (46). [Note that in this case the last term on the righthand side of Eq. (54) is of order $a^{3} / R^{3}$ and, thus, should be disregarded in the framework of the PFA.]

It is easily seen that two additional terms on the righthand side of Eq. (54) are negligibly small. For example, for the same parameters $R=L=100 \mu \mathrm{m}$, as earlier, and $L_{1}=$ $25 \mu \mathrm{m}$ the contribution of these terms does not exceed $10^{-4} \%$ at $a=100 \mathrm{~nm}$ and $0.05 \%$ at $a=500 \mathrm{~nm}$. With the increase of $L_{1}$ the contribution of the additional terms in Eq. (54) further decreases. For instance, for $L_{1}=50 \mu \mathrm{m}$ it does not exceed $5 \times 10^{-7} \%$ at $a=100 \mathrm{~nm}$ and $1.5 \times 10^{-4} \%$ at $a=500 \mathrm{~nm}$.

\section{PROPOSED EXPERIMENTAL SETUP}

In this section we propose an experiment to measure the Casimir interaction between a cylinder and a plate using an approach similar to what was used in previous studies for sphere-plate geometry [32-36,70]. A sensitive micromechanical torsional oscillator (MTO) is at the heart of the setup since it allows detection of the Casimir force in a very precise manner. An incorporated optical fiber two-color interferometer allows the measurement of the fiber-to-substrate separation at all times. As was done in our previous experiments, an electrostatic calibration will allow us to determine the interaction force, as well as the separation $a$.

We propose to integrate a metallic cylinder onto a MTO and to detect the deflections experienced by the micromechanical device when a metallic plate approaches the cylinder from above (see Fig. 4 for a schematic of the setup). Section II presents theoretical results for the Casimir force and for the gradient of the Casimir force between a cylinder and a plate. 


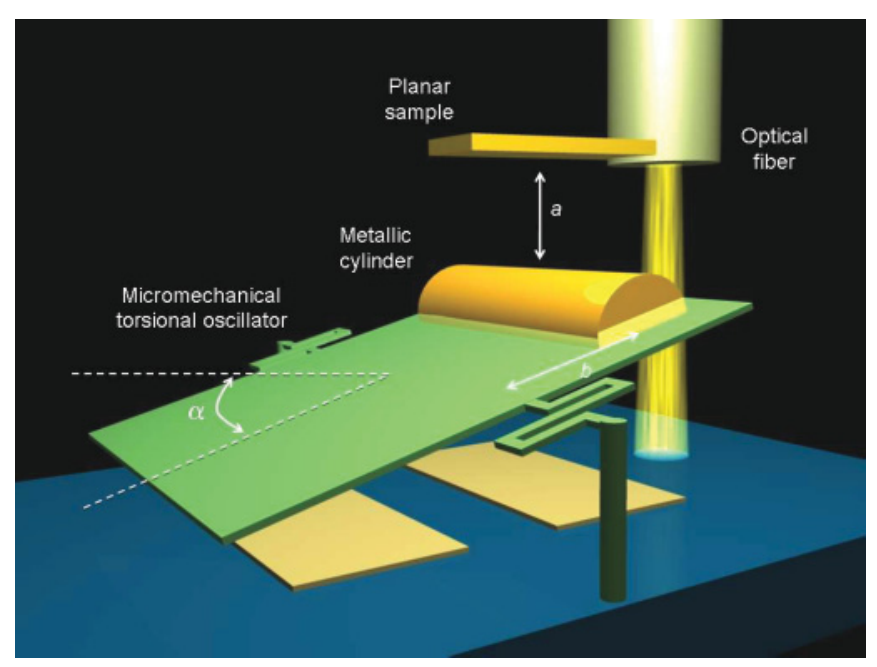

FIG. 4. (Color online) Schematic of the experimental setup (see text for further discussion). The figure is not to scale.

Both these quantities can be measured using the suggested setup. The Casimir force is measured in the static mode [32]. The gradient of the Casimir force is measured in the dynamic mode, where the separation distance between a cylinder and a plate is varied harmonically at the resonant frequency of the MTO [33]. The experimental setup allows for a force sensitivity of $\sim 0.1 \mathrm{pN}$ and a determination of the resonant frequency of the MTO to better than $6 \mathrm{mHz}$. In the dynamic mode, the gradient of the Casimir force is obtained from the resonant frequency of the oscillator:

$$
\omega_{\text {res }}=\omega_{0}\left(1-\frac{b^{2}}{2 I \omega_{0}^{2}} \frac{\partial F}{\partial a}\right) .
$$

Here $\omega_{0}$ is the natural angular resonance frequency of the oscillator, $\omega_{\text {res }}$ is the angular resonant frequency of the oscillator in the presence of the Casimir force, $b$ is the lever arm of the interaction, and $I$ is the moment of inertia of the oscillator.

In order to minimize errors associated with misalignments between the torsional axis of the MTO and the cylinder's axis, we propose to integrate the metallic cylinder onto the MTO using state-of-the-art semiconducting processing. Currently available lithographic steppers allow alignment between different structural layers in a mechanical device with an accuracy of $20 \mathrm{~nm}$ or better. By using a monolithic fabrication process it would be possible to directly fabricate a metallic cylinder on top of a MTO similar to the one previously used. In order to fabricate a cylinder with a predetermined cylindrical shape and smooth surface, it will be necessary to use grayscale masks or laser-based maskless lithography technology [71]. These technologies permit the creation of three-dimensional structures with smooth surfaces and precise critical dimensions. A simpler method of fabricating a cylindrical object on top of a MTO involves the deposition and patterning of photosensitive polymers (photoresist) and subsequent thermal reflow of the polymer into a curved shape. This technique relies on surface tension and plastic flow of photoresist and is used to produce smooth curved objects. Reflow techniques are much simpler to implement than grayscale lithography techniques, but they are much more limited regarding the final shape of the cylindrical objects. The photoresist shape can be transferred to the MTO using conventional dry etching techniques like reactive ion etching (RIE). Once the cylindrical shape has been transferred to the MTO, it can be coated with a metallic film by evaporation or sputtering methods.

The actual cylindrical shape $z=f(x, y)$ can be measured very precisely, better than $1 \mathrm{~nm}$ error in $z$, by using a noncontact optical profilometer. Once this shape is known, it can be introduced in Eq. (1) to calculate the Casimir force acting on the cylinder, as described in Sec. II.

During the measurements, the cylinder-MTO structure will be brought close to the Au-coated plane using a five-axis manipulator with an angular error of $10^{-5} \mathrm{rad}$. This angular error allows for an alignment of the cylinder and the plate to within the ranges calculated in Sec. IV.

Further improvements in the alignment between the axis of the MTO and the plate can be obtained by sacrificing the ability to perform continuous measurements as a function of separation. In this approach the oscillator or cylinder unit is manufactured as previously described, but the Au-coated plate on a $\mathrm{Si}$ substrate is engineered with three equal pillars of known height. The MTO chip is positioned on top of the pillars and a measurement of the Casimir interaction at a fixed separation can be performed. By changing the height of the pillars, it is feasible to reproduce a curve of the Casimir interaction as a function of separation. Since the height of the pillars can be controlled to better than $10 \mathrm{~nm}$, misalignments smaller than $1 \mu \mathrm{rad}$ are possible.

\section{CONCLUSIONS AND DISCUSSION}

In the foregoing we have investigated the possibilities of measuring the thermal Casimir force and its gradient between a plate and a microfabricated cylinder attached to a micromachined oscillator. Using the PFA we have derived the Lifshitz-type formulas for the thermal Casimir force for a cylinder and a plate made of real materials. From the comparison with exact results available for an ideal metal cylinder above a plate and for both ideal and real metals in the configuration of a sphere above a plate, we have estimated the error resulting from the use of the PFA. It turns out that for reasonable experimental parameters this error does not exceed a small fraction of a percent. In the limiting case of high temperature (large separation) the analytic expressions for the Casimir force and its gradient are obtained.

The derived Lifshitz-type formulas were used to perform numerical computations of the thermal correction to both the Casimir force and its gradient. In so doing the two alternative theoretical approaches proposed in the literature (the Drude model and the plasma model approaches) have been used. The predicted results of both static and dynamic experiments for the measurement of the thermal correction were discussed. Specifically, it was concluded that the thermal correction, as predicted by the Drude model approach, is large enough to be measurable at short separations below $1 \mu \mathrm{m}$. With respect to the thermal correction predicted by the plasma model approach, it can be measured only at separations of a few micrometers. The performed computations, when compared with the experimental data, may shed additional light on 
the experimental exclusion of the Drude model approach in Refs. [32-36] which was made using only the configuration of a sphere above a plate.

Keeping in mind the applications of the developed formalism for comparison with experimental data, we have derived expressions for the thermal Casimir force and its gradient taking into account the nonparallelity of the cylinder and plate. This was done in a nonmultiplicative manner including the role of correlations between geometrical and material properties. In the specific case of ideal metal bodies at zero temperature, the previously obtained results were confirmed. We have also performed numerical computations of a correction to the Casimir force due to nonparallelity of a cylinder and a plate using both nonmultiplicative and multiplicative approaches and determined the application region of the latter.

Taking into account that microfabricated cylinders to be used in experiments are restricted in length (which may be of the order of the cylinder radius), we have estimated errors in computations arising from the finiteness of a cylinder. The two experimentally relevant cases were considered when the cylinder is completely over the plate or is partially beyond the plate edge. In both cases it was shown that for typical experimental parameters the total error due to application of the PFA and due to edge effects is sufficiently small and does not depend on the ratio of the cylinder radius to the cylinder length. This opens opportunities for using cylinders with $L \approx$ $R$, with no loss in the accuracy of the theoretical expressions derived for infinitely long cylinders.

We have presented a scheme of the proposed experiment for measuring thermal Casimir force and its gradient in the configuration of an Au-coated plate and a microfabricated cylinder attached to the micromachined oscillator. Both the static and the dynamic versions of this experiment are considered. The values of main experimental parameters that are sought are estimated. The proposed experiment is promising as a source of additional information on the problem of thermal Casimir force.

\section{ACKNOWLEDGMENTS}

R.S.D. acknowledges NSF support through Grant No. PHY0701236 and LANL support through Contract No. 49423001-07. D.L. and R.S.D. acknowledge support from DARPA Grant No. 09-Y557. E.F. was supported in part by the DOE under Grant No. DE-76ER071428. G.L.K. and V.M.M. are grateful to the Department of Physics, Purdue University, for financial support. G.L.K. was also partially supported by Russian Ministry of Education Grant No. P-184.
[1] H. B. G. Casimir, Proc. K. Ned. Akad. Wet. B 51, 793 (1948).

[2] V. M. Mostepanenko and N. N. Trunov, The Casimir Effect and Its Applications (Clarendon, Oxford, 1997).

[3] P. W. Milonni, The Quantum Vacuum (Academic Press, San Diego, 1994).

[4] M. Krech, The Casimir Effect in Critical Systems (World Scientific, Singapore, 1994).

[5] K. A. Milton, The Casimir Effect (World Scientific, Singapore, 2001).

[6] M. Bordag, G. L. Klimchitskaya, U. Mohideen, and V. M. Mostepanenko, Advances in the Casimir Effect (Oxford University Press, Oxford, 2009).

[7] E. M. Lifshitz, Zh. Eksp. Teor. Fiz. 29, 94 (1956) [Sov. Phys. JETP 2, 73 (1956)].

[8] I. E. Dzyaloshinskii, E. M. Lifshitz, and L. P. Pitaevskii, Usp. Fiz. Nauk 73, 381 (1961) [Adv. Phys. 10, 165 (1961)].

[9] S. K. Lamoreaux, Phys. Rev. Lett. 78, 5 (1997).

[10] U. Mohideen and A. Roy, Phys. Rev. Lett. 81, 4549 (1998).

[11] G. L. Klimchitskaya, U. Mohideen, and V. M. Mostepanenko, Rev. Mod. Phys. 81, 1827 (2009).

[12] V. B. Bezerra, G. L. Klimchitskaya, and V. M. Mostepanenko, Phys. Rev. A 65, 052113 (2002); 66, 062112 (2002).

[13] V. B. Bezerra, G. L. Klimchitskaya, V. M. Mostepanenko, and C. Romero, Phys. Rev. A 69, 022119 (2004).

[14] B. Geyer, G. L. Klimchitskaya, and V. M. Mostepanenko, Phys. Rev. D 72, 085009 (2005).

[15] B. Geyer, G. L. Klimchitskaya, and V. M. Mostepanenko, Int. J. Mod. Phys. A 21, 5007 (2006).

[16] B. Geyer, G. L. Klimchitskaya, and V. M. Mostepanenko, Ann. Phys. (NY) 323, 291 (2008).

[17] M. Bordag and I. Pirozhenko (to appear in Phys. Rev. D), e-print arXiv:1010.1217.

[18] M. Boström and B. E. Sernelius, Physica A 339, 53 (2004).
[19] I. Brevik, J. B. Aarseth, J. S. Høye, and K. A. Milton, Phys. Rev. E 71, 056101 (2005).

[20] J. S. Høye, I. Brevik, S. A. Ellingsen, and J. B. Aarseth, Phys. Rev. E 75, 051127 (2007).

[21] I. Brevik, S. A. Ellingsen, J. S. Høye, and K. A. Milton, J. Phys. A 41, 164017 (2008).

[22] L. P. Pitaevskii, Phys. Rev. Lett. 101, 163202 (2008).

[23] D. A. R. Dalvit and S. K. Lamoreaux, Phys. Rev. Lett. 101, 163203 (2008).

[24] B. Geyer, G. L. Klimchitskaya, U. Mohideen, and V. M. Mostepanenko, Phys. Rev. Lett. 102, 189301 (2009).

[25] L. P. Pitaevskii, Phys. Rev. Lett. 102, 189302 (2009).

[26] R. S. Decca, E. Fischbach, B. Geyer, G. L. Klimchitskaya, D. E. Krause, D. López, U. Mohideen, and V. M. Mostepanenko, Phys. Rev. Lett. 102, 189303 (2009).

[27] D. A. R. Dalvit and S. K. Lamoreaux, Phys. Rev. Lett. 102, 189304 (2009).

[28] V. M. Mostepanenko, J. Phys. Conf. Ser. 161, 012003 (2009).

[29] G. L. Klimchitskaya, J. Phys. Conf. Ser. 161, 012002 (2009).

[30] D. A. R. Dalvit and S. K. Lamoreaux, J. Phys. Conf. Ser. 161, 012009 (2009)

[31] V. M. Mostepanenko, R. S. Decca, E. Fischbach, B. Geyer, G. L. Klimchitskaya, D. E. Krause, D. López, and U. Mohideen, Int. J. Mod. Phys. A 24, 1721 (2009).

[32] R. S. Decca, E. Fischbach, G. L. Klimchitskaya, D. E. Krause, D. López, and V. M. Mostepanenko, Phys. Rev. D 68, 116003 (2003).

[33] R. S. Decca, D. López, E. Fischbach, G. L. Klimchitskaya, D. E. Krause, and V. M. Mostepanenko, Ann. Phys. (NY) 318, 37 (2005).

[34] G. L. Klimchitskaya, R. S. Decca, E. Fischbach, D. E. Krause, D. López, and V. M. Mostepanenko, Int. J. Mod. Phys. A 20, 2205 (2005). 
[35] R. S. Decca, D. López, E. Fischbach, G. L. Klimchitskaya, D. E. Krause, and V. M. Mostepanenko, Phys. Rev. D 75, 077101 (2007).

[36] R. S. Decca, D. López, E. Fischbach, G. L. Klimchitskaya, D. E. Krause, and V. M. Mostepanenko, Eur. Phys. J. C 51, 963 (2007).

[37] F. Chen, G. L. Klimchitskaya, V. M. Mostepanenko, and U. Mohideen, Opt. Express 15, 4823 (2007).

[38] F. Chen, G. L. Klimchitskaya, V. M. Mostepanenko, and U. Mohideen, Phys. Rev. B 76, 035338 (2007).

[39] J. M. Obrecht, R. J. Wild, M. Antezza, L. P. Pitaevskii, S. Stringari, and E. A. Cornell, Phys. Rev. Lett. 98, 063201 (2007).

[40] G. L. Klimchitskaya and V. M. Mostepanenko, J. Phys. A 41, 312002(F) (2008).

[41] G. Bressi, G. Carugno, R. Onofrio, and G. Ruoso, Phys. Rev. Lett. 88, 041804 (2002).

[42] V. M. Mostepanenko and G. L. Klimchitskaya, Int. J. Mod. Phys. A 25, 2302 (2010).

[43] D. A. R. Dalvit, F. C. Lombardo, F. D. Mazzitelli, and R. Onofrio, Europhys. Lett. 67, 517 (2004).

[44] M. Brown-Hayes, D. A. R. Dalvit, F. D. Mazzitelli, W. J. Kim, and R. Onofrio, Phys. Rev. A 72, 052102 (2005).

[45] T. Emig, R. L. Jaffe, M. Kardar, and A. Scardicchio, Phys. Rev. Lett. 96, 080403 (2006).

[46] M. Bordag, Phys. Rev. D 73, 125018 (2006).

[47] F. C. Lombardo, F. D. Mazzitelli, and P. I. Villar, Phys. Rev. D 78, 085009 (2008).

[48] W. J. Kim, M. Brown-Hayes, D. A. R. Dalvit, J. H. Brownell, and R. Onofrio, Phys. Rev. A 78, 020101(R) (2008).

[49] W. J. Kim, A. O. Sushkov, D. A. R. Dalvit, and S. K. Lamoreaux, Phys. Rev. A 81, 022505 (2010).

[50] Q. Wei, D. A. R. Dalvit, F. C. Lombardo, F. D. Mazzitelli, and R. Onofrio, Phys. Rev. A 81, 052115 (2010).

[51] R. S. Decca, E. Fischbach, G. L. Klimchitskaya, D. E. Krause, D. López, U. Mohideen, and V. M. Mostepanenko, Phys. Rev. A 79, 026101 (2009).
[52] H. Gies and K. Klingmüller, Phys. Rev. Lett. 97, 220405 (2006).

[53] B. V. Derjaguin, Kolloidn. Zh. 69, 155 (1934).

[54] J. Błocki, J. Randrup, W. J. Swiatecki, and C. F. Tsang, Ann. Phys. (NY) 105, 427 (1977).

[55] R. S. Decca, E. Fischbach, G. L. Klimchitskaya, D. E. Krause, D. López, and V. M. Mostepanenko, Phys. Rev. D 79, 124021 (2009).

[56] M. Bordag and I. Pirozhenko, Phys. Rev. D 81, 085023 (2010).

[57] A. Canaguier-Durand, P. A. Maia Neto, I. Cavero-Pelaez, A. Lambrecht, and S. Reynaud, Phys. Rev. Lett. 102, 230404 (2009).

[58] P. A. Maia Neto, A. Lambrecht, and S. Reynaud, Phys. Rev. A 78, 012115 (2008).

[59] T. Emig, J. Stat. Mech. (2008) P04007.

[60] R. Zandi, T. Emig, and U. Mohideen, Phys. Rev. B 81, 195423 (2010).

[61] A. Canaguier-Durand, P. A. Maia Neto, A. Lambrecht, and S. Reynaud, Phys. Rev. Lett. 104, 040403 (2010).

[62] A. Canaguier-Durand, P. A. Maia Neto, A. Lambrecht, and S. Reynaud, Phys. Rev. A 82, 012511 (2010).

[63] E. D. Palik ed., Handbook of Optical Constants of Solids (Academic Press, New York, 1985).

[64] A. Lambrecht and S. Reynaud, Eur. Phys. J. D 8, 309 (2000).

[65] G. L. Klimchitskaya, U. Mohideen, and V. M. Mostepanenko, J. Phys. A: Math. Gen. 40, F339 (2007).

[66] B. Geyer, G. L. Klimchitskaya, and V. M. Mostepanenko, J. Phys. A 40, 13485 (2007).

[67] G. Bimonte, Phys. Rev. A 81, 062501 (2010).

[68] B. Geyer, G. L. Klimchitskaya, and V. M. Mostepanenko, Phys. Rev. B 81, 245421 (2010).

[69] S. G. Rabinovich, Measurement Errors and Uncertainties. Theory and Practice (Springer-Verlag, New York, 2000).

[70] R. S. Decca, D. López, E. Fischbach, and D. E. Krause, Phys. Rev. Lett. 91, 050402 (2003).

[71] H. P. Herzig ed., Micro-Optics: Elements, Systems and Applications (Taylor and Francis, Washington, 1997). 